(Respond: Dr Shevell, Room A-514, Montreal Children's Hoispital, 2300 Tupper Street, Montreal, Quebec H3H IP3, Canada).

COMMENT. The authors list further investigations to identify the cause of spastic diplegia in the high proportion $(46 \%)$ of patients undiagnosed. These investigations include repeat MRI, spinal imaging, voxel-based morphometry, and diffusion-weighted MRI in the acute stage of PVL.

\title{
THIRD VENTRICLE ENLARGEMENT IN NEONATES WITH TRISOMY 21
}

Measurements of routine head sonographic scans of 57 term infants with trisomy 21 born between 2000 and 2005 were performed within 7 days after birth and were compared with scans of 21 randomly selected, healthy, term infants without trisomy 21 at Shaare Zedek Medical Center, Jerusalem; Ben Gurion University of the Negev, Beer Sheva; and Hadassah University Hospital, Jerusalem, Israel. The test and control neonates were the same gestational ages (39+/-1 weeks), but trisomy 21 infants were smaller and had smaller head circumferences than controls $(32.9 \mathrm{~cm}$ vs $34.9 \mathrm{~cm} ; \mathrm{P}=0.001)$. The width and length of the third ventricle were increased in infants with trisomy 21 . Vertical measurements of the lateral ventricle were similar for the 2 groups. (Schimmel MS, Hammerman C, Bromiker R, Berger I. Third ventricle enlargement among newborn infants with trisomy 21. Pediatrics May 2006;117:928-931). (Respond: Michael S Schimmel MD, Department of Neonatology, Shaare Zedek Medical Center, PO Box 3235, Jerusalem 91031, Israel).

COMMENT. The authors speculate that the enlargement of the third ventricle demonstrated in neonates with trisomy 21 may reflect hypoplasia of the thalamus, hypothalamus, and deep white matter, which are involved in cognitive processes of attention, verbal and visuospatial memory. Prefrontal, cerebellar, and hippocampal functions are also affected in trisomy 21 .

\section{SERUM BILIRUBIN LEVELS AND DEVELOPMENTAL OUTCOME}

The neurodevlopmental risks associated with neonatal total serum bilirubin levels of $25 \mathrm{mg} / \mathrm{dL}$ or higher in 140 affected infants were compared with 419 randomly selected controls from a cohort of term-infants born 1995-1998 in Kaiser Permanente hospitals in northern California. Peak bilirubin levels were between 25 and $29.9 \mathrm{mg} / \mathrm{dL}$ in 130 newborns with hyperbilirubinemia and $30 \mathrm{mg} / \mathrm{dL}$ or higher in 10 newborns. Phototherapy was used in 136 cases and exchange transfusion in 5. There were no cases of kernicterus. In subjects followed for at least 2 years, scores on cognition tests were similar in the hyperbilirubinemia and control groups. Questionable or abnormal neurologic findings were present in 14 children (17\%) with hyperbilirubinemia vs 48 of controls $(29 \%) ; \mathrm{P}=0.05$. Reported behavioral problems were not significantly different in the 2 groups. Those with positive direct antiglobulin tests for immune-mediated hemolytic disease in the hyperbilirubinemia group had lower scores on cognition tests but not more neurologic or behavioral problems. (Newman TB, Liljestrand P, Jeremy RJ, et al. Outcomes among newborns with total serum 
bilirubin levels of $25 \mathrm{mg}$ per deciliter or more. N Engl J Med May 4, 2006;354:1889-1900). (Respond: Dr Thomas B Newman, Kaiser Permanente Medical Center, Oakland, CA).

COMMENT. When treated with phototherapy or exchange transfusion, neonates born at or near term and having a total serum bilirubin of $25-30 \mathrm{mg} / \mathrm{dL}$ for less than 6 hours showed no increase in neurodevelopmental or behavioral problems at follow-up compared to controls. In an editorial, Watchko JF (N Engl J Med May 4, 2006;354:1947-1948) points out that factors other than serum bilirubin level and coexisting hemolysis can increase the risk of kernicterus: reduced albumin binding of bilirubin, low gestational age, glucose-6-phosphate dehydrogenase deficiency, and acidosis. These factors may lower the serum bilirubin level at which treatment is indicated. In 116 reported cases of kernicterus, 90 had total serum bilirubin levels of $30 \mathrm{mg} / \mathrm{dL}$ or more and 76 had levels of $35 \mathrm{mg} / \mathrm{dL}$ or more.

\section{NEUROMUSCULAR DISORDERS}

\section{SEVERITY AND OUTCOME OF MYASTHENIA GRAVIS}

Myasthenia gravis (MG) severity and long-term prognosis in seronegative, seropositive, and thymoma MG were studied retrospectively in 4 previously reported series of age and sex-matched consecutive patients at Haukeland University Hospital, University of Bergen, Norway. Patients were assessed annually and were followed for 30 to 40 years. Fifty-two early onset cases ( 34 thymectomized and 18 non-thymectomized) were followed for 39 years. Thymectomized patients showed remissions in $21 / 34$, whereas only $4 / 18$ nonthymectomized patients had remission. The presence of AChR antibodies correlates with a more severe MG. With proper treatment, especially early thymectomy for seropositive MG, the outcome and long-term prognosis is good in patients with or without $\mathrm{AChR}$ antibodies. Thymectomy is of benefit in early-onset MG, but not in late-onset MG. In early-onset MG, thymectomy should be considered soon after onset, and outcome is not influenced by muscle autoantibodies. In 43 late-onset cases, MG severity did not differ between thymectomized ( 21 cases) and non-thymectomized patients (12 cases). Thymoma ( 24 cases) and nonthymoma (24) MG have equal severity and outcome of MG. Ryanodine receptor (RyR) antibodies in thymoma MG and titin/RyR in non-thymoma MG may indicate a less favorable outcome. (Romi F, Gilhus NE, Aarli JA. Myasthenia gravis: disease severity and prognosis. Acta Neurol Scand May 2006;113 (Suppl 183):24-25). (Respond: Dr Fredrik Romi, Department of Neurology, Haukeland University Hospital, Bergen, Norway).

COMMENT. Thymectomy is of benefit in early onset MG but not in late-onset MG. In a study of $51 \mathrm{MG}$ patients (35 juvenile cases) at the Massachusetts General Hospital, 18 $(86 \%)$ of 21 who underwent thymectomy showed complete or partial remission (Millichap JG, Dodge PR. Neurology 1960;10:1007-1014). Of 14 patients in this series who were treated with medication alone, $13(93 \%)$ obtained remission, and initial results were comparable to those treated surgically. Remission was sustained in only $2(14 \%)$ of drug treated vs $6(29 \%)$ thymectomized patients. The earlier the surgery, the better chance of remission (Andrews PI et al. Neurology 1994;44:1208-1214).

Arthrogryposis multiplex congenita (AMC) is reported in 3 infants (including twins) born to a mother with MG. (Hoff JM et al. Acta Neurol Scand May 2006;113 (Suppl 183):26-27). 